\title{
Comprehensive Analysis of miRNA-mRNA Regulatory Network And Potential Drugs In Chronic Chagasic Cardiomyopathy Across Human And Mouse
}

\author{
Jiahe Wu \\ Zhongnan Hospital of Wuhan University \\ Jianlei Cao \\ Zhongnan Hospital of Wuhan University \\ Yongzhen Fan \\ Zhongnan Hospital of Wuhan University \\ Chenze Li \\ Zhongnan Hospital of Wuhan University \\ Xiaorong Hu ( $\nabla$ huxrzn@whu.edu.cn ) \\ Zhongnan Hospital of Wuhan University
}

\section{Research Article}

Keywords: Chronic chagasic cardiomyopathy, Hub Gene, miRNA-mRNA regulatory network, Gene function enrichment analysis, PPI Network, Bioinformatics analysis

Posted Date: September 15th, 2021

DOI: https://doi.org/10.21203/rs.3.rs-835491/v1

License: (c) (1) This work is licensed under a Creative Commons Attribution 4.0 International License.

Read Full License

Version of Record: A version of this preprint was published at BMC Medical Genomics on November 29th, 2021. See the published version at https://doi.org/10.1186/s12920-021-01134-3. 


\section{Abstract}

Background: Chronic chagasic cardiomyopathy (CCC) is the leading cause of heart failure in Latin America and often causes severe inflammation and fibrosis in the heart. Studies on myocardial function and its molecular mechanisms in patients with Chronic chagasic cardiomyopathy are very limited. In order to understand the development and progression of Chronic chagasic cardiomyopathy and find targets for its diagnosis and treatment, the field needs to better understand the exact molecular mechanisms involved in these processes.

Methods: The mRNA microarray datasets GSE84796 (human) and GSE24088 (mouse) were obtained from the Gene Expression Omnibus (GEO) database. Homologous genes between the two species were identified using the online database mining tool Biomart, followed by differential expression analysis, gene enrichment analysis and protein-protein interaction (PPI) network construction. Cytohubba plug-in of Cytoscape software was used to identify Hub gene, and miRNet was used to construct the corresponding miRNA-mRNA regulatory network. Furthermore, Comparative Toxicogenomics Database (CTD) was used to identify hub gene-related drugs.

Results: A total of 86 homologous genes were significantly differentially expressed in the two datasets, including 73 genes with high expression and 13 genes with low expression. These differentially expressed genes were mainly enriched in the terms of innate immune response, signal transduction, protein binding, Natural killer cell mediated cytotoxicity, Tuberculosis, Chemokine signaling pathway, Chagas disease and PI3K-Akt signaling pathway. The top 10 hub genes LAPTM5, LCP1, HCLS1, COR01A, CD48, TYROBP, RAC2, ARHGDIB, FERMT3 and NCF4 were identified from the PPI network. A total of 122 miRNAs were identified to target these hub genes and 30 of them regulated two or more hub genes at the same time. Finally, 49 drugs, such as Gentamicins, acetamide, Isotretinoin, Nimesulide, Oxyquinoline, Quercetin and Resveratrol were identified to target these hub genes.

Conclusions: In this study, the potential genes associated with Chronic chagasic cardiomyopathy are identified and a miRNA-mRNA regulatory network is constructed. This study explores the molecular mechanisms of Chronic chagasic cardiomyopathy and provides important clues for finding new therapeutic targets.

\section{Introduction}

Chronic Chagasic Cardiomyopathy (CCC), first reported by Carlos Chagas in 1909, is a myocardial disease caused by a protozoan parasite, Trypanosoma cruzi[1, 2]. CCC is characterized by severe cardiac inflammation and myocardial fibrosis and is the leading cause of myocardial diseases and heart failure in Latin America [3]. The incidence of CCC has declined as living conditions have improved, but the current trend of people moving from rural to urban areas complicates the situation[4]. Studies have shown that chagas disease still affects 6 to 8 million people and kills about 12,000 people a year[5] and 
the positive rate of T. cruzi in serum of Latin American immigrants with non-ischemic cardiomyopathy in the United States ranges from 13-19\%[6].

CCC often causes symptoms of palpitations or syncope from arrhythmia, chest pain from microvascular dysfunction and heart failure from left ventricular dysfunction.

The pathogenesis of chronic chagas cardiomyopathy is very complex. At present, there are four hypotheses: direct injury caused by parasites[7], inflammatory reaction[8], microvascular injury[9] and autonomic nerve dysfunction[10]. Patients eventually died of malignant arrhythmia (55\%-65\%) in early adulthood, mainly because persistent ventricular tachycardia (VT) degenerated into ventricular fibrillation[11]. There are many studies on CCC, but there is still no effective treatment at present, and the prognosis of chagas cardiomyopathy is very poor.

Animal models of CCC, especially mouse models, have attracted researchers' attention due to the high homologous and identity of human and mouse genome[12, 13]. The research on the similarity of the molecular characteristics of human and mouse diseases has led to the rapid development of disease prognosis and treatment. Studies have shown that RARP1 plays an important role in CCC, which leads to the increase of mtROS and oxidative stress in chagasic myocardium through the decrease of Pol $Y^{-}$ dependent mtDNA content, mtDNA coding gene expression and mitochondrial respiratory function[14]. This indicates that RARP1 may be an important therapeutic target of CCC. Furthermore, TGF- $\beta$ inhibitor therapy can decreases fibrosis and stimulates cardiac improvement in CCC[15]. The dysregulation of microRNAs (miRNAs), including: miR-19a-3p, miR-21-5p, miR29b-3p, miR-21, miR-146 are reported to be related with CCC development[16-18]. However, the identity of miRNA-mRNA regulations in CCC had not been reported till now.

This study was performed to identify the co-differently expressed miRNA-mRNA regulatory networks and developmental programs in CCC between human and mouse. The GSE84796 and GSE24088 datasets were downloaded and homologous genes between human and mouse CCC models were identified and analyzed using bioinformatics analyses. Our study would provide new insights of molecular mechanisms involved CCC, aiming to understand the development and progression of CCC and find targets for its diagnosis and treatment. The workflow of the specific analysis is shown in Fig. 1.

\section{Methods}

\section{Data resource}

The mRNA microarray datasets GSE84796 (human) and GSE24088 (mouse) were obtained from the Gene Expression Omnibus (GEO) database. (https://www.ncbi.nlm.nih.gov/). GSE84796 (human) and GSE24088 (mouse) is based on the GPL14550 platform (Agilent-028004; SurePrint G3 Human GE 8x60K Microarray) and the GPL8938 platform (Duke Mouse 30k; Oligonucleotide Array Operon V3.0.1). GSE84796 is comprised of $10 \mathrm{CCC}$ samples (patients have severe Chagas Chronic Cardiomyopathy) and 7 control samples (healthy organ donors, these hearts were not used for transplantation due to size 
mismatch with available recipients). GSE24088 is comprised of 4 CCC samples (C57BI/6 mice chronically infected with T. cruzi (6 months)) and 4 control samples (normal control).

\section{Identification of DEGs in Chronic Chagasic Cardiomyopathy}

The gene expression dataset was downloaded from the abovementioned platform and GEO2R was adopted to identify the DEGs between CCC samples and control samples. The adjusted P-values (adj. P) and log-fold change (FC) in expression were determined. Benjamini-hochberg method with default values was used to correct adj. P for potential false positive results. Adj. $\mathrm{P}<0.05$ and $|\log \mathrm{FC}|>1.0$ was set as the specific cut-off criteria of DEGs.

\section{Annotations of Homologous Genes Across Species}

The homologous genes between human and mouse were identified using the online database mining tool Biomart[19, 20]. (v2.3.6; https://bioconductor.org/packages/release/bioc/html/biomaRt.html)

\section{Identification of Homologous DEGs Between Human and Mouse}

Venn diagram web tool (http://bioinformatics.psb.ugent.be/webtools/Venn/) was used and the identified homologous genes were intersected with genes that had obvious common differential expression in the two datasets. The heat maps of Homologous DEGs were produced using the package g plots of $\mathrm{R}$ software (version: x64 3.2.1)[21, 22].

\section{Function Enrichment Analysis of Homologous DEGs}

The Gene Ontology (GO; http://www.geneontology.org) knowledgebase is the world's largest source of information on the functions of genes. GO describes the function of gene products in all organisms and identify transcriptome data or characteristic biological properties of high-throughput genomes. Three categories: biological process (BP), cellular component (CC) and molecular function (MF) are its main classification[23]. Kyoto Encyclopedia of Genes and Genomes (KEGG;

http://www.kegg.jp/orhttp://www.genome.jp/kegg/) is a database resource for understanding high-level functions and utilities of the biological system, such as the cell, the organism and the ecosystem, from molecular-level information, especially large-scale molecular datasets generated by genome sequencing and other high-throughput experimental technologies[24]. The Database for Annotation, Visualization, and Integrated Discovery (DAVID; version6.8; https://david.ncifcrf.gov) was used for enrichment analysis of Homologous DEGs. P value $<0.05$ was regarded as statistically significant.

\section{Construction of the Protein-Protein Interaction Network and Module analysis}

In order to better understand the molecular mechanism of CCC, a protein-protein interaction network (PPI network) of Homologous DEGs was constructed. The String database (version10.0; http://string-db.org) was used to perform critical assessments and integrations of protein interactions, including physical (direct) and functional (indirect) associations[25]. Based on the results of the analysis, PPI network was 
established by Cytoscape software (version 3.7.1). Taken the scores of Maximal Clique Centrality (MCC) algorithm as the criteria, the Cytohubba plug-in of Cytoscape software was used to screen out the top10 Hub genes with high connectivity in the PPI network. What's more, the significant modules of the PPI network were screened using the plug-in MCODE (the parameters were set to default). The KOBAS (version 3.0; http://kobas.cbi.pku.edu.cn/) database was used to explore the key pathways of these modules[26].

\section{Construction of the miRNA-Hub Gene regulatory network}

miRNet (https://www.mirnet.ca) is an easy-to-use, miRNA-centric network visual analytics platform aiming to help elucidate microRNA functions by integrating existing knowledge with users' data [27]. miRNet was used to identify miRNAs targeting Hub gene. The miRNA-mRNA network of CCC was established by Cytoscape software (version 3.7.1).

\section{Hub Gene-Drug Interaction Network Analysis}

The public Comparative Toxicogenomics Database (CTD; http://ctdbase.org/) is a digital ecosystem that relates toxicological information for chemicals, genes, phenotypes, diseases [28]. The hub gene-drug interaction network was constructed using CTD, aiming to search chemotherapeutic drugs that could reduce or increase the expression levels of the hub genes. Briefly, these hub genes were searched in CTD database, and the hub gene-drug interaction networks were visualized by Cytoscape software (version 3.7.1).

\section{Results}

\section{Identification of Homologous DEGs Between Human and Mouse}

The mRNA microarray datasets GSE84796 (human) and GSE24088 (mouse) were obtained from the Gene Expression Omnibus (GEO) database. According to the GEO2R software analysis and the screening conditions, DEGs in each GEO dataset were identified. There were 1615 DEGs, including 1165 upregulated and 450 downregulated genes in GSE84796 (human), 1032 DEGs, including 789 upregulated and 243 downregulated genes in GSE24088 (mouse). The volcano plots of the distribution of DEGs in each dataset are shown in Fig. 2A-B. The DEGs are listed in Additional file 1. The top 10 high-expressed DEGs and the top 10 low-expressed DEGs in each dataset are shown in Table 1-2. The online database mining tool Biomart was used to identify the homologous genes. A total of 17354 homologous genes were identified and these homologous genes between human and mouse are list in Additional file 2. These identified homologous genes were intersected with genes that had obvious common differential expression in the two datasets and 86 Homologous DEGs (Additional file 3), including 73 upregulated and 13 downregulated genes, were identified. Figure $2 C-D$ is the Venn diagram showing this process. Figure 2E is the Heatmap of these Homologous DEGs in GSE84796 (human). 
Table 1

The top 10 high-expressed DEGs and the top 10 low-expressed DEGs in GSE84796

\begin{tabular}{|llllll|}
\hline Datasete & GENE_SYMBOL & gene ID & logFC & adj.P.Value & expression \\
\hline GSE84796 (human) & IGLL5 & 100423062 & 9.3527332 & $5.72 \mathrm{E}-08$ & up \\
\cline { 2 - 6 } & SCML4 & 256380 & 7.8225719 & $3.85 \mathrm{E}-08$ & up \\
\hline IGLL1 & 3543 & 7.0467008 & $2.07 \mathrm{E}-08$ & up \\
\hline POU2AF1 & 5450 & 6.7202013 & $6.84 \mathrm{E}-07$ & up \\
\hline MZB1 & 51237 & 6.6000016 & $1.38 \mathrm{E}-07$ & up \\
\hline CCL5 & 6352 & 6.2834279 & $8.89 \mathrm{E}-07$ & up \\
\hline IGJ & 3512 & 6.183125 & $1.66 \mathrm{E}-08$ & up \\
\hline GZMH & 2999 & 6.1234204 & $6.47 \mathrm{E}-08$ & up \\
\hline ZNF683 & 257101 & 6.0890868 & $5.72 \mathrm{E}-08$ & up \\
\hline FCRL5 & 83416 & 6.0100721 & $5.72 \mathrm{E}-08$ & up \\
\hline ELF3 & 1999 & -5.2432822 & $3.15 \mathrm{E}-02$ & down \\
\hline RPS4Y1 & 6192 & -5.1884702 & $7.90 \mathrm{E}-02$ & down \\
\hline RPS4Y2 & 140032 & -5.1431484 & $7.72 \mathrm{E}-02$ & down \\
\hline NCRNA00185 & 83869 & -4.605292 & $4.00 \mathrm{E}-02$ & down \\
\hline DDX3Y & 8653 & -4.5198597 & $5.46 \mathrm{E}-02$ & down \\
\hline BMP7 & 655 & -3.9880484 & $7.84 \mathrm{E}-04$ & down \\
\hline D4S234E & 27065 & -3.7308209 & $1.16 \mathrm{E}-05$ & down \\
\hline PCDH20 & 64881 & -3.6182641 & $1.44 \mathrm{E}-03$ & down \\
\hline EIF1AY & 9086 & -3.4750827 & $7.78 \mathrm{E}-02$ & down \\
\hline AGXT2L1 & 64850 & -3.447762 & $2.28 \mathrm{E}-05$ & down \\
\hline
\end{tabular}


Table 2

The top 10 high-expressed DEGs and the top 10 low-expressed DEGs in GSE24088

\begin{tabular}{|c|c|c|c|c|c|}
\hline Datasete & GENE_SYMBOL & gene ID & $\log \mathrm{FC}$ & adj.P.Value & expression \\
\hline \multirow[t]{20}{*}{ GSE24088 (mouse) } & Ccl8 & 20307 & 5.441605 & 7.70E-04 & up \\
\hline & Timp1 & 21857 & 5.429127 & $6.84 \mathrm{E}-04$ & up \\
\hline & Pla2g7 & 27226 & 5.320059 & $6.84 \mathrm{E}-04$ & up \\
\hline & Ctss & 13040 & 5.296175 & $1.20 \mathrm{E}-03$ & up \\
\hline & $\mathrm{Cfb}$ & 14962 & 5.233954 & $1.21 \mathrm{E}-03$ & up \\
\hline & $\mathrm{H} 2-\mathrm{Aa}$ & 14960 & 5.064829 & 2.35E-03 & up \\
\hline & Plac8 & 231507 & 4.932353 & $6.84 \mathrm{E}-04$ & up \\
\hline & Lilrb4 & 292594 & 4.907422 & $1.20 \mathrm{E}-03$ & up \\
\hline & Itgb1bp3 & 69564 & 4.834374 & 2.07E-03 & up \\
\hline & Mpeg1 & 17476 & 4.831578 & 1.99E-03 & up \\
\hline & Ces3 & 23491 & -2.746517 & 2.62E-02 & down \\
\hline & Sucla2 & 20916 & -2.321407 & 1.01E-02 & down \\
\hline & Acat1 & 110446 & -2.195231 & $3.50 \mathrm{E}-02$ & down \\
\hline & Efnb3 & 13643 & -2.164247 & 9.97E-03 & down \\
\hline & Mkks & 59030 & -2.121421 & $2.38 \mathrm{E}-02$ & down \\
\hline & Dnaja3 & 83945 & -2.120821 & $4.23 \mathrm{E}-03$ & down \\
\hline & Lrtm1 & 319476 & -2.076317 & $1.51 \mathrm{E}-02$ & down \\
\hline & Gapdh & 14433 & -2.068512 & 3.50E-02 & down \\
\hline & Asb15 & 78910 & -2.030904 & 4.46E-02 & down \\
\hline & 3110057012Rik & 269423 & -1.992578 & 3.49E-02 & down \\
\hline
\end{tabular}

\section{Enrichment Analysis for Homologous DEGs}

GO knowledgebase and KEGG database were used to characterize the functional roles of the above Homologous DEGs. Figure 3 list the top 15 enriched $G 0$ terms and KEGG pathways $(p<0.05)$. The BP category of the GO analysis results showed that these Homologous DEGs were significantly enriched in the term of innate immune response, leukocyte migration, signal transduction, positive regulation of cell migration and adaptive immune response (Fig. 3A). For GO CC analysis, the top four significantly enriched terms were cytosol, phagocytic vesicle membrane, actin filament, lamellipodium (Fig. 3B). The top four significantly enriched MF terms included Toll - like receptor binding, actin binding, protein 
binding, SH3 domain binding (Fig. 3C). Furthermore, Natural killer cell mediated cytotoxicity, Platelet activation, Tuberculosis, Leukocyte transendothelial migration, Chemokine signaling pathway, PI3K - Akt signaling pathway and Chagas disease (American trypanosomiasis) are pathways of significant enrichment in KEGG analysis (Fig. 3D). Detailed analysis results are in Additional file 4.

\section{Construction of the Protein-Protein Interaction Network and identification of Hub Genes and Key Modules}

The Homologous DEGs obtained were introduced into the online database String. After removing the isolated genes without interaction, a PPI network with 72 nodes and 279 edges was established (Fig. 4A). The 11 yellow nodes in the network represent down-regulated genes and the 61 blue nodes in the network represent up-regulated genes. Next, we filtered out the top 10 hub genes using the plug-in Cytohubba in Cytoscape using MCC method. They were LAPTM5, LCP1, HCLS1, CORO1A, CD48, TYROBP, RAC2, ARHGDIB, FERMT3 and NCF4. The PPI network of these 10 hub genes is shown in (Fig. 4B). We identified 3 modules in the whole network on the basis of MCODE (Fig. 4C-E).

Pathway enrichment analysis shows that these 10 hub genes are significantly enriched in the pathway of Natural killer cell mediated cytotoxicity (Fig. 5). In this pathway diagram, the Hub gene is highlighted by a subscript red segment. In addition, the DEGs of module 1 are significantly enriched in the pathway of Natural killer cell mediated cytotoxicity, Leukocyte transendothelial migration, Platelet activation and Phagosome. The DEGs of module 2 are enriched in Prion diseases, Fc epsilon RI signaling pathway, Staphylococcus aureus infection and Pertussis. Furthermore, the DEGs of module 3 are significantly enriched in Fc epsilon RI signaling pathway, B cell receptor signaling pathway, Fc gamma R-mediated phagocytosis and Natural killer cell mediated cytotoxicity. These hub gene and genes of top 3 modules are shown in Table 3.

Table 3

The top three significant modules and hub genes in the PPI network

\begin{tabular}{|c|c|c|c|c|}
\hline Plug-in & Modules & Nodes & Edges & Genes \\
\hline MCODE & $\begin{array}{l}\text { Module } \\
1\end{array}$ & 15 & 89 & $\begin{array}{l}\text { RAC2, FERMT3, ARHGAP9, LSP1, CORO1A, GMFG, } \\
\text { TYROBP, LAPTM, CD 52, CD 48, NCF4, LCP1, ARHGDIB, } \\
\text { HCLS1, APBB1IP }\end{array}$ \\
\hline MCODE & $\begin{array}{l}\text { Module } \\
2\end{array}$ & 5 & 9 & C1QA, CCR5, ALOX5AP, MPEG1, AIF1 \\
\hline MCODE & $\begin{array}{l}\text { Module } \\
3\end{array}$ & 5 & 9 & DOK2, INPP5D, JAK3, SYK, PTK2B \\
\hline CytoHubba & $\begin{array}{l}\text { Hub } \\
\text { gene }\end{array}$ & 10 & 45 & $\begin{array}{l}\text { LAPTM5, LCP1, HCLS1, COR01A, CD 48, TYROBP, RAC2, } \\
\text { ARHGDIB, FERMT3, NCF4 }\end{array}$ \\
\hline
\end{tabular}

\section{Construction of the miRNA-Hub Gene regulatory network}


miRNet was used to identify miRNAs targeting Hub genes and a miRNA-mRNA regulatory network with 132 nodes (122miRNAs and 10 hub genes) and 170 edges was established (Fig. 6A). We screened miRNAs that simultaneously targeted two or more Hub genes and constructed another miRNA-mRNA regulatory network with 40 nodes (30miRNAs and 10 hub genes) and 78 edges (Fig. 6B). MiRNAs of hsamiR-34a-5p, hsa-miR-30d-5p, hsa-miR-27a-3p, hsa-miR-24-3p, hsa-miR-203a-3p, hsa-miR-16-5p, hsa-miR155-5p, hsa-miR-1-3p, hsa-miR-129-2-3p and hsa-miR-124-3p, which targeted 3 or more Hub genes, are screened (Additional file 5). Hsa-miR-34a-5p and hsa-miR-155-5p targets six hub genes simultaneously. We identified these correlations using two miRNA-related online databases, Targetscan (version7.2; targetscan.org)[29] and miRDB ( http://mirdb.org)[30], and identified 8 regulatory relationships: has-miR34a-5p-ARHGDIB, has-miR-34c-5p-ARHGDIB, has-miR-16-5p-LCP1, has-miR-34b-5p-LCP1, has-miR-124-3pLCP1, has-miR-424-5p-LCP1, has-miR-129-2-3p-HCLS1, has-miR-107-HCLS1.

\section{Drug-Hub Gene Interaction Network Analysis}

Bennidazole or nifurtimox in the treatment of trypanosomiasis is the standard treatment for Chagas' disease and has been shown to be effective in the acute phase of human trials. However, a large, prospective, multicentre, randomized study of 2854 patients with CCC in which bennidazole was used to interrupt the trypanosomiasis evaluation (BENEFIT) test was negative, showing that bennidazole did not significantly reduce chronic cardiac clinical deterioration[31]. Therefore, it is very meaningful to find new drugs that can change the prognosis of CCC.

The hub genes were inserted into CTD and the targeted drugs that could reduce or increase the expression levels of these genes were predicted. Finally, a total of 272 target drugs were predicted. We screened drugs that simultaneously targeted three or more Hub genes and constructed a drug-hub gene interaction network with 59 nodes (49 drugs and 10 hub genes) and 249 edges (Fig. 7). For example, LCP1, COR01A, RAC2, ARHGDIB, FERMT3 and NCF4 are down-regulated but CD48, HCLS1, LAPTM5 and TYROBP are up-regulated by Antirheumatic Agents. Gentamicins can target 8 hub genes, CD48, HCLS1, LCP1, RAC2 are up regulated and ARHGDIB, COR01A, LAPTM5, TYROBP are down-regulated. Quercetin can target ARHGDIB, HCLS1, LAPTM5 and down-regulated them. In addition, Acetamide, Isotretinoin, Nimesulide, Oxyquinoline and Resveratrol were identified to target these hub genes.

\section{Discussion}

CCC is an inflammatory dilated cardiomyopathy caused by a protozoan parasite, Trypanosoma cruzi [32]. In the past hundred years, a large number of studies have been conducted on CCC. However, the research on the molecular mechanism of $\mathrm{CCC}$ and the effective treatment methods are very limited. We use bioinformatics method to conduct data mining on CCC-related datasets, extract relevant biological information from high-dimensional data, and find the key pathways and hub genes that affect CCC occurrence. This method is the new trend of precision medical era disease research. These identified molecules have the potential to be used as disease biomarkers for patient-specific treatment and to improve the diagnosis, treatment, and prevention of CCC. 
In the present study, we screened 86 Homologous genes including 73 upregulated and 13 downregulated genes which were significantly differentially expressed in the two datasets across human and mouse. GO and KEGG pathway enrichment analysis showed that these Homologous DEGs were significantly enriched in immune-related functions and pathways. These genes functions in immune-related biological processes such as innate immune response, leukocyte migration, signal transduction, adaptive immune response, phagocytic vesicle membrane, Toll - like receptor binding and protein binding. Furthermore, these Homologous DEGs are involved in Natural killer cell mediated cytotoxicity, Leukocyte transendothelial migration, Chemokine signaling pathway, PI3K - Akt signaling pathway and Chagas disease (American trypanosomiasis). The PPI network of these Homologous DEGs was analyzed by String and Visualized by Cytoscape software. Based on analyzing of this network, 10 hub genes, LAPTM5, LCP1, HCLS1, COR01A, CD48, TYROBP, RAC2, ARHGDIB, FERMT3 and NCF4 were identified. These genes are enriched in the pathway of Natural killer cell mediated cytotoxicity.

Several studies had been published about Natural killer cell mediated cytotoxicity pathway in CCC. Killer cell-mediated parasite death, which known as "microbiological programmed cell death" is similar to mammalian apoptosis and results in membrane potential decomposition, vesiculation, phospholipserine exposure, mitochondrial swelling, chromatin condensation, and DNA damage. Antimicrobial peptide granulysin (GNLY), pore-forming perforin (PFN) and granzyme (GZM) can eliminate intracellular protozoan parasites by Natural killer cell mediated cytotoxicity pathway [33]. It has been shown that infection with a virulent T. cruzi strain alters NK cell-mediated regulation of adaptive immune responses induced by DC cells[34]. Therefore, by intervening in this pathway, it is possible to eliminate pathogens infected cells and delay the progression of CCC. What's more, Leukocyte transendothelial migration[35], Chemokine signaling pathway[36] and PI3K - Akt signaling pathway[37] may also be key pathways to the regulation of CCC.

As for hub genes, many studies have linked them to cardiomyopathy. For example, a genome-wide association study identifies the functional relationships between the associated variant and FERMT3 (Fermitin family homolog 3) in CCC[38]. TYROBP (TYRO protein tyrosine kinase-binding protein) shows significant increasing expression levels in model HCM (Hypertrophic cardiomyopathy) rats and affected the immune system significantly[39]. NCF4 (Neutrophil cytosol factor 4) is the component of the NADPHoxidase. RAC2 (Ras-related C3 botulinum toxin substrate 2) can augment the production of reactive oxygen species (ROS) by NADPH oxidase. L7DG (luteolin-7-diglucuronide) pretreatment can block ISOstimulated expression of the NCF4 and RAC2, protecting the heart against developing ISO-induced injury and fibrosis[40]. CD48 is the ligand of CD2, may facilitate interaction between activated lymphocytes and involve in regulating T-cell activation[41]. LCP1 (L-plastin, also known as plastin-2) binds to aMß2 integrin and maintains its inactivity, thus regulating leukocyte adhesion to integrin ligand in the flow state[42]. These studies suggest that these hub genes are involved in the process of inflammatory response and myocardial remodeling and may play an important role in the development of CCC.

miRNAs are endogenous approximately 23nt RNAs that pairing with mRNAs of protein-coding genes to guide their post-transcriptional suppression[43]. These hub genes have reported to be a direct target of 
several miRNAs including miR-125a-5p[44], miR-375[45], miR-125[46],miR-183[47]. Based on these hub genes, miRNAs (such as hsa-miR-34a-5p, hsa-miR-30d-5p, hsa-miR-27a-3p, hsa-miR-24-3p, hsa-miR-203a$3 p$, hsa-miR-16-5p, hsa-miR-155-5p, hsa-miR-1-3p, hsa-miR-129-2-3p and hsa-miR-124-3p) that may regulate $\mathrm{CCC}$ were predicted by miRNet tool. Many studies have linked these miRNAs to cardiomyopathy. Inhibition of miR-34a in mice attenuates moderate cardiac dysfunction by inhibiting atrial expandatrial enlargement[48]. miR-30 participates in ventricular remodeling through autophagy, apoptosis, oxidative stress and inflammation[49]. Mir-24-3p regulates KEAR1-NRF2 pathway and protects myocardial cells after ischemia/reperfusion injury in mice[50]. These results demonstrated the potential roles of these miRNAs in regulating inflammation and fibrosis of the heart in CCC.

At present, Bennidazole or nifurtimox is the main treatment for Chagas' disease. However, there is currently no effective treatment for CCC. A total of 50 Drugs (such as Gentamicins, acetamide, Isotretinoin, nimesulide, Oxyquinoline, Quercetin and Resveratrol) that may regulate CCC were predicted by CTD database. These drugs can protect the heart by regulating inflammation, autophagy, apoptosis and other pathways[51-53]. These drugs can serve as a starting point for follow-up studies.

The analysis of high differentially expressed homologous genes in human and mouse is helpful to understand the common characteristics and pathogenesis of CCC among species. Further experiments to verify the functions of these genes will give a more realistic picture of what happens in the human body. However, this study is based on published datasets and has some limitations. Further in vivo and in vitro studies, as well as large sample multicenter clinical studies, are required to explore better diagnostic and therapeutic methods for CCC.

\section{Conclusions}

In conclusion, we identified there were 86 homologous DEGs in CCC between human and mouse. Bioinformatics analyses showed 122 miRNAs and 10 hub genes were associated with CCC pathogenesis via "Natural killer cell mediated cytotoxicity, Chemokine signaling pathway, Chagas disease and PI3K Akt signaling pathway" pathways. A total of 49 drugs were predicted and may be candidates for the treatment of CCC. However, the present study is a preliminary analysis, and further studies, both in vivo and in vitro, are needed to confirm these insights.

\section{Abbreviations}

CCC Chronic Chagasic Cardiomyopathy

GEO database Gene Expression Omnibus database.

DEG Differently expressed genes

GO Gene Ontology 
BP Biological process

CC Cellular component

MF Molecular function

KEGG Kyoto Encyclopedia of Genes and Genomes

DAVID Database for Annotation, Visualization, and Integrated Discovery

PPI network Protein-Protein interaction network

MCC Maximal Clique Centrality

CTD Comparative Toxicogenomics Database

NC Natural killer

DC Dendritic cells

GNLY Granulysin

PFN Pore-forming perforin

GZM Granzyme

HCM Hypertrophic cardiomyopathy

\section{Declarations}

Ethical approval and consent to participate

This article does not contain any studies with human participants or animals performed by any of the authors. Therefore, ethical approval and informed consent are not required.

Consent for publication

Not applicable.

Availability of data and materials

The following information was supplied regarding data availability: Data is available at NCBI GEO: GSE84796 and GSE24088

Competing interests

The authors declare that they have no conflicts of interest. 


\section{Funding}

This study was supported by a grant from The National Natural Science foundation of China (No.81370308, Xiaorong Hu) and a grant from Zhongnan Hospital of Wuhan University Science, Technology and Innovation Seed Fund (N0. znpy2018099, Xiaorong Hu) and the research fund from medical Sci-Tech innovation platform of Zhongnan Hospital, Wuhan University (No. ptxm2021009, Jianlei Cao)

\section{Authors' Contributions}

Jiahe Wu performed the data analysis and drafted the manuscript.

Yongzhen Fan and Chenze Li prepared the figures and contributed towards the study design.

Xiaorong Hu and Jianlei Cao revised the figures, designed and supervised the study.

All authors have read and approved the final manuscript.

\section{Acknowledgments}

We sincerely appreciate the researchers for providing their GEO database information online, we are truly honored to acknowledge their contributions.

\section{Online Database address:}

Gene Expression Omnibus (GEO) database. (https://www.ncbi.nlm.nih.gov/)

Biomart (v2.3.6; https://bioconductor.org/packages/release/bioc/html/biomaRt.html)

Venn diagram web tool (http://bioinformatics.psb.ugent.be/webtools/Venn/)

Gene Ontology (GO; http://www.geneontology.org)

KEGG (http://www.kegg.jp/orhttp://www.genome.jp/kegg/)

DAVID (version6.8; https://david.ncifcrf.gov)

String database (version10.0; http://string-db.org)

KOBAS (version 3.0; http://kobas.cbi.pku.edu.cn/)

miRNet (https://www.mirnet.ca)

CTD (http://ctdbase.org/)

miRDB ( http://mirdb.org) 
Targetscan (version7.2; http://targetscan.org)

\section{References}

1. Rassi, A.J., A. Rassi and J.A. Marin-Neto, Chagas disease. Lancet, 2010. 375(9723): p. 1388-402.

2. Bern, C., Chagas' Disease. N Engl J Med, 2015. 373(5): p. 456-66.

3. Velasco, A. and C.A. Morillo, Chagas heart disease: A contemporary review. J Nucl Cardiol, 2020. 27(2): p. 445-451.

4. Traina, M.I., et al., Prevalence and Impact of Chagas Disease Among Latin American Immigrants With Nonischemic Cardiomyopathy in Los Angeles, California. Circ Heart Fail, 2015. 8(5): p. 938-43.

5. Bonney, K.M., et al., Pathology and Pathogenesis of Chagas Heart Disease. Annu Rev Pathol, 2019. 14: p. 421-447.

6. Kapelusznik, L., et al., Chagas disease in Latin American immigrants with dilated cardiomyopathy in New York City. Clin Infect Dis, 2013. 57(1): p. e7.

7. Cunha-Neto, E., et al., Induction of cardiac autoimmunity in Chagas heart disease: a case for molecular mimicry. Autoimmunity, 2006. 39(1): p. 41-54.

8. Mangini, S., et al., Infectious agents and inflammation in donated hearts and dilated cardiomyopathies related to cardiovascular diseases, Chagas' heart disease, primary and secondary dilated cardiomyopathies. Int J Cardiol, 2015. 178: p. 55-62.

9. Marin-Neto, J.A., et al., Pathogenesis of chronic Chagas heart disease. Circulation, 2007. 115(9): p. 1109-23.

10. Villar, J.C., H. Leon and C.A. Morillo, Cardiovascular autonomic function testing in asymptomatic T. cruzi carriers: a sensitive method to identify subclinical Chagas' disease. Int J Cardiol, 2004. 93(23): p. 189-95.

11. Echavarria, N.G., et al., Chagas Disease: Chronic Chagas Cardiomyopathy. Curr Probl Cardiol, 2021. 46(3): p. 100507.

12. Rada, J., et al., IL-10-Dependent and -Independent Mechanisms Are Involved in the Cardiac Pathology Modulation Mediated by Fenofibrate in an Experimental Model of Chagas Heart Disease. Front Immunol, 2020. 11: p. 572178.

13. Santos, E.S., et al., Tolerogenic Dendritic Cells Reduce Cardiac Inflammation and Fibrosis in Chronic Chagas Disease. Front Immunol, 2020. 11: p. 488.

14. Wen, J.J., Y.W. Yin and N.J. Garg, PARP1 depletion improves mitochondrial and heart function in Chagas disease: Effects on POLG dependent mtDNA maintenance. PLoS Pathog, 2018. 14(5): p. e1007065.

15. Ferreira, R.R., et al., TGF-beta inhibitor therapy decreases fibrosis and stimulates cardiac improvement in a pre-clinical study of chronic Chagas' heart disease. PLoS Negl Trop Dis, 2019. 13(7): p. e0007602. 
16. Nonaka, C., et al., Circulating miRNAs as Potential Biomarkers Associated with Cardiac Remodeling and Fibrosis in Chagas Disease Cardiomyopathy. Int J Mol Sci, 2019. 20(16).

17. Nonaka, C., et al., Therapeutic miR-21 Silencing Reduces Cardiac Fibrosis and Modulates Inflammatory Response in Chronic Chagas Disease. Int J Mol Sci, 2021. 22(7).

18. Ballinas-Verdugo, M.A., et al., Circulating miR-146a as a possible candidate biomarker in the indeterminate phase of Chagas disease. Biol Res, 2021. 54(1): p. 21.

19. Smedley, D., et al., The BioMart community portal: an innovative alternative to large, centralized data repositories. Nucleic Acids Res, 2015. 43(W1): p. W589-98.

20. Durinck, S., et al., BioMart and Bioconductor: a powerful link between biological databases and microarray data analysis. Bioinformatics, 2005. 21(16): p. 3439-40.

21. Ritchie, M.E., et al., limma powers differential expression analyses for RNA-sequencing and microarray studies. Nucleic Acids Res, 2015. 43(7): p. e47.

22. Gentleman, R.C., et al., Bioconductor: open software development for computational biology and bioinformatics. Genome Biol, 2004. 5(10): p. R80.

23. Gene Ontology Consortium: going forward. Nucleic Acids Res, 2015. 43(Database issue): p. D104956.

24. Kanehisa, M., et al., KEGG: new perspectives on genomes, pathways, diseases and drugs. Nucleic Acids Res, 2017. 45(D1): p. D353-D361.

25. von Mering, C., et al., STRING: a database of predicted functional associations between proteins. Nucleic Acids Res, 2003. 31(1): p. 258-61.

26. Shannon, P., et al., Cytoscape: a software environment for integrated models of biomolecular interaction networks. Genome Res, 2003. 13(11): p. 2498-504.

27. Chang, L., et al., miRNet 2.0: network-based visual analytics for miRNA functional analysis and systems biology. Nucleic Acids Res, 2020. 48(W1): p. W244-W251.

28. Davis, A.P., et al., Comparative Toxicogenomics Database (CTD): update 2021. Nucleic Acids Res, 2021. 49(D1): p. D1138-D1143.

29. Agarwal, V., et al., Predicting effective microRNA target sites in mammalian mRNAs. Elife, 2015. 4.

30. Chen, $Y$. and $X$. Wang, miRDB: an online database for prediction of functional microRNA targets. Nucleic Acids Res, 2020. 48(D1): p. D127-D131.

31. Morillo, C.A., et al., Randomized Trial of Benznidazole for Chronic Chagas' Cardiomyopathy. N Engl J Med, 2015. 373(14): p. 1295-306.

32. Laugier, L., et al., miRNAs may play a major role in the control of gene expression in key pathobiological processes in Chagas disease cardiomyopathy. PLoS Negl Trop Dis, 2020. 14(12): p. e0008889.

33. Dotiwala, F., et al., Killer lymphocytes use granulysin, perforin and granzymes to kill intracellular parasites. Nat Med, 2016. 22(2): p. 210-6. 
34. Batalla, E.I., et al., Impairment in natural killer cells editing of immature dendritic cells by infection with a virulent Trypanosoma cruzi population. J Innate Immun, 2013. 5(5): p. 494-504.

35. Panagiotidou, S., et al., Trypanosoma cruzi Exploits E- and P-Selectins to Migrate Across Endothelial Cells and Extracellular Matrix Proteins. Infect Immun, 2021: p. IAI0017821.

36. Gil-Jaramillo, N., et al., The First Contact of Human Dendritic Cells With Trypanosoma cruzi Reveals Response to Virus as an Unexplored Central Pathway. Front Immunol, 2021. 12: p. 638020.

37. Penas, F.N., et al., Pyridinecarboxylic Acid Derivative Stimulates Pro-Angiogenic Mediators by $\mathrm{PI3K} / \mathrm{AKT} / \mathrm{mTOR}$ and Inhibits Reactive Nitrogen and Oxygen Species and NF-kappaB Activation Through a PPARgamma-Dependent Pathway in T. cruzi-Infected Macrophages. Front Immunol, 2019. 10: p. 2955.

38. Casares-Marfil, D., et al., A genome-wide association study identifies novel susceptibility loci in chronic Chagas cardiomyopathy. Clin Infect Dis, 2021.

39. Chen, R., et al., Identification of biomarkers correlated with hypertrophic cardiomyopathy with coexpression analysis. J Cell Physiol, 2019. 234(12): p. 21999-22008.

40. Ning, B.B., et al., Luteolin-7-diglucuronide attenuates isoproterenol-induced myocardial injury and fibrosis in mice. Acta Pharmacol Sin, 2017. 38(3): p. 331-341.

41. Lissina, A., et al., Fine-tuning of CD8(+) T-cell effector functions by targeting the 2B4-CD48 interaction. Immunol Cell Biol, 2016. 94(6): p. 583-92.

42. Tseng, H.Y., et al., LCP1 preferentially binds clasped alphaMbeta2 integrin and attenuates leukocyte adhesion under flow. J Cell Sci, 2018. 131(22).

43. Bartel, D.P., MicroRNAs: target recognition and regulatory functions. Cell, 2009. 136(2): p. 215-33.

44. Bhatlekar, S., et al., miR-125a-5p regulates megakaryocyte proplatelet formation via the actinbundling protein L-plastin. Blood, 2020. 136(15): p. 1760-1772.

45. Jimenez, L., et al., miR-375 Regulates Invasion-Related Proteins Vimentin and L-Plastin. Am J Pathol, 2017. 187(7): p. 1523-1536.

46. Wojtowicz, E.E., et al., MicroRNA-125 family members exert a similar role in the regulation of murine hematopoiesis. Exp Hematol, 2014. 42(10): p. 909 - 18.e1.

47. Donatelli, S.S., et al., TGF-beta-inducible microRNA-183 silences tumor-associated natural killer cells. Proc Natl Acad Sci U S A, 2014. 111(11): p. 4203-8.

48. Bernardo, B.C., et al., Silencing of miR-34a attenuates cardiac dysfunction in a setting of moderate, but not severe, hypertrophic cardiomyopathy. PLoS One, 2014. 9(2): p. e90337.

49. Zhang, X., et al., The microRNA in ventricular remodeling: the miR-30 family. Biosci Rep, 2019. 39(8).

50. Xiao, X., et al., MicroRNA miR-24-3p Reduces Apoptosis and Regulates Keap1-Nrf2 Pathway in Mouse Cardiomyocytes Responding to Ischemia/Reperfusion Injury. Oxid Med Cell Longev, 2018. 2018: p. 7042105.

51. Booth, E.A., et al., Estrogen protects the heart from ischemia-reperfusion injury via COX-2-derived PGI2. J Cardiovasc Pharmacol, 2008. 52(3): p. 228-35. 
52. Mascareno, E., et al., Rapidly progressive course of Trypanosoma cruzi infection in mice heterozygous for hexamethylene bis-acetamide inducible 1 (Hexim1) gene. Microbes Infect, 2018. 20(1): p. 25-36.

53. Wu, X., et al., Autophagy and cardiac diseases: Therapeutic potential of natural products. Med Res Rev, 2021. 41(1): p. 314-341.

Figures 


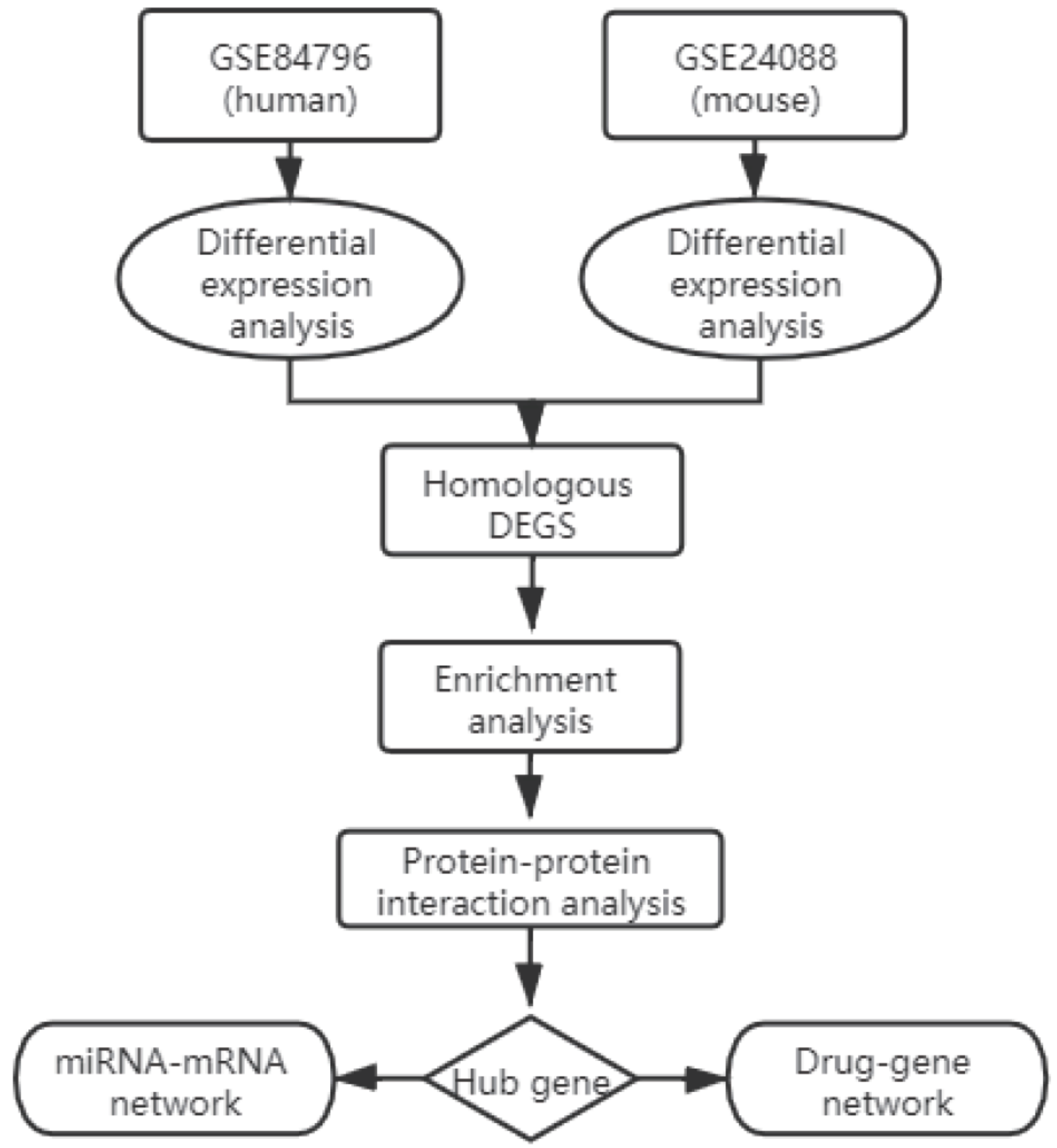

Figure 1

Flowchart of the steps performed in this study 
A

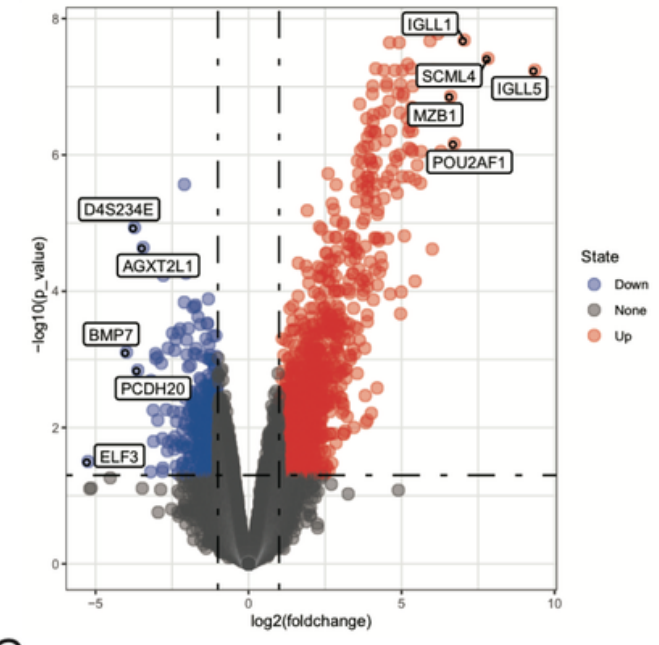

C

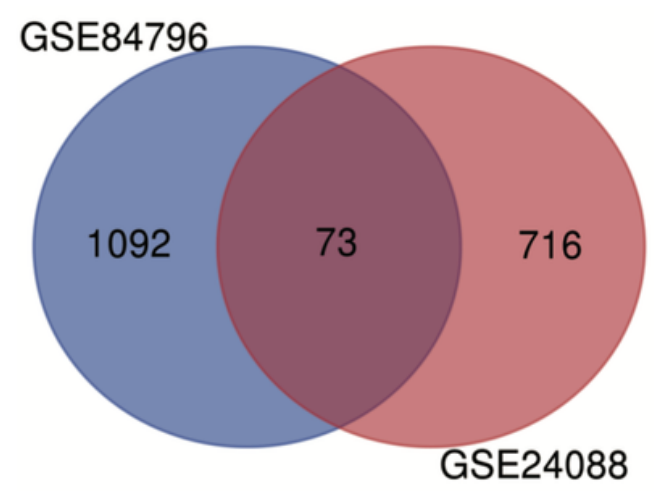

B

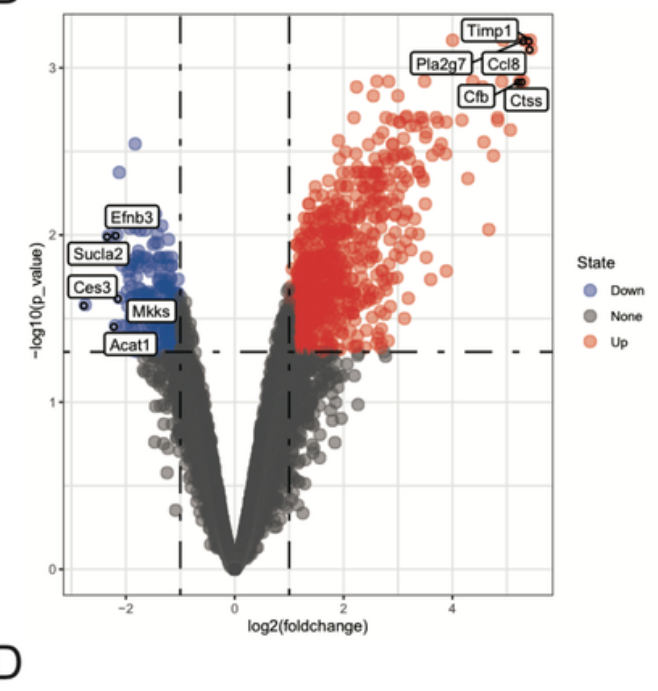

GSE84796

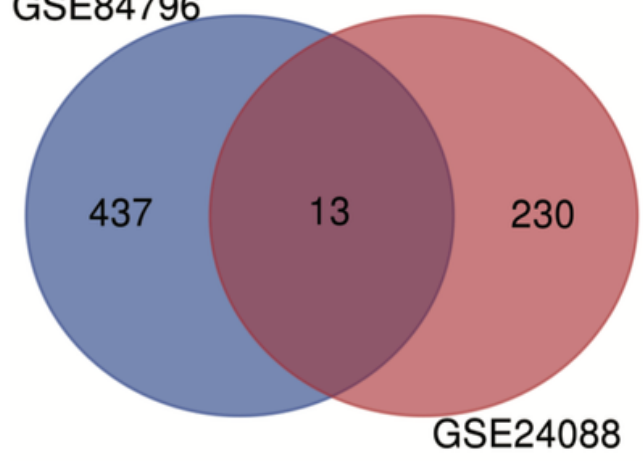

$E$

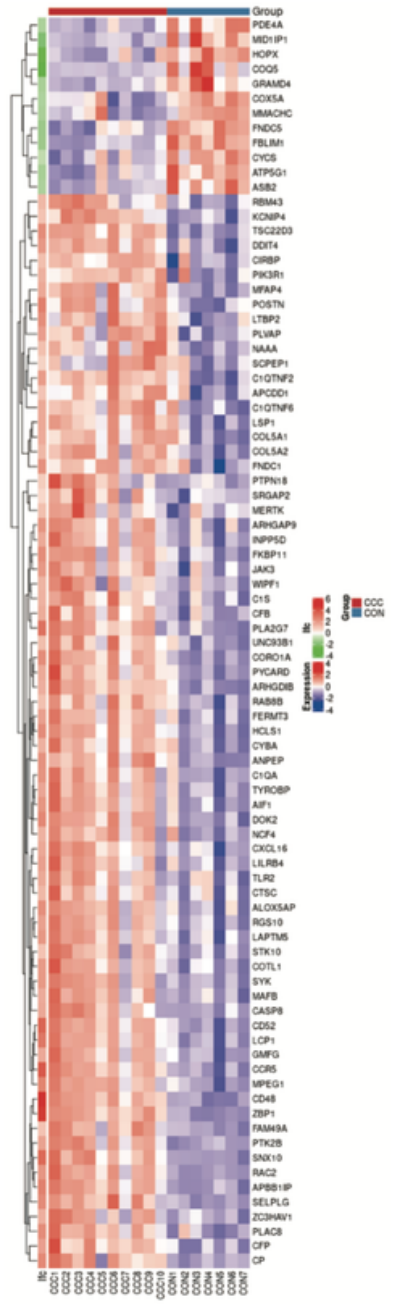

Figure 2

Homologous DEGs in GSE84796 (human) and GSE24088 (mouse) (A)Volcano plots of GSE84796. (B) Volcano plots of GSE24088. For A and B, differentially expressed molecules were screened under the cutoff criteria $|\log 2 \mathrm{FC}|>1$ and the adjusted P-value $(P<0.05)$. (C) Venn diagram of up-regulated Homologous DEGs. (D) Venn diagram of down-regulated homologous DEGs. (E) Heatmap of Homologous DEGs in GSE84796. 
A

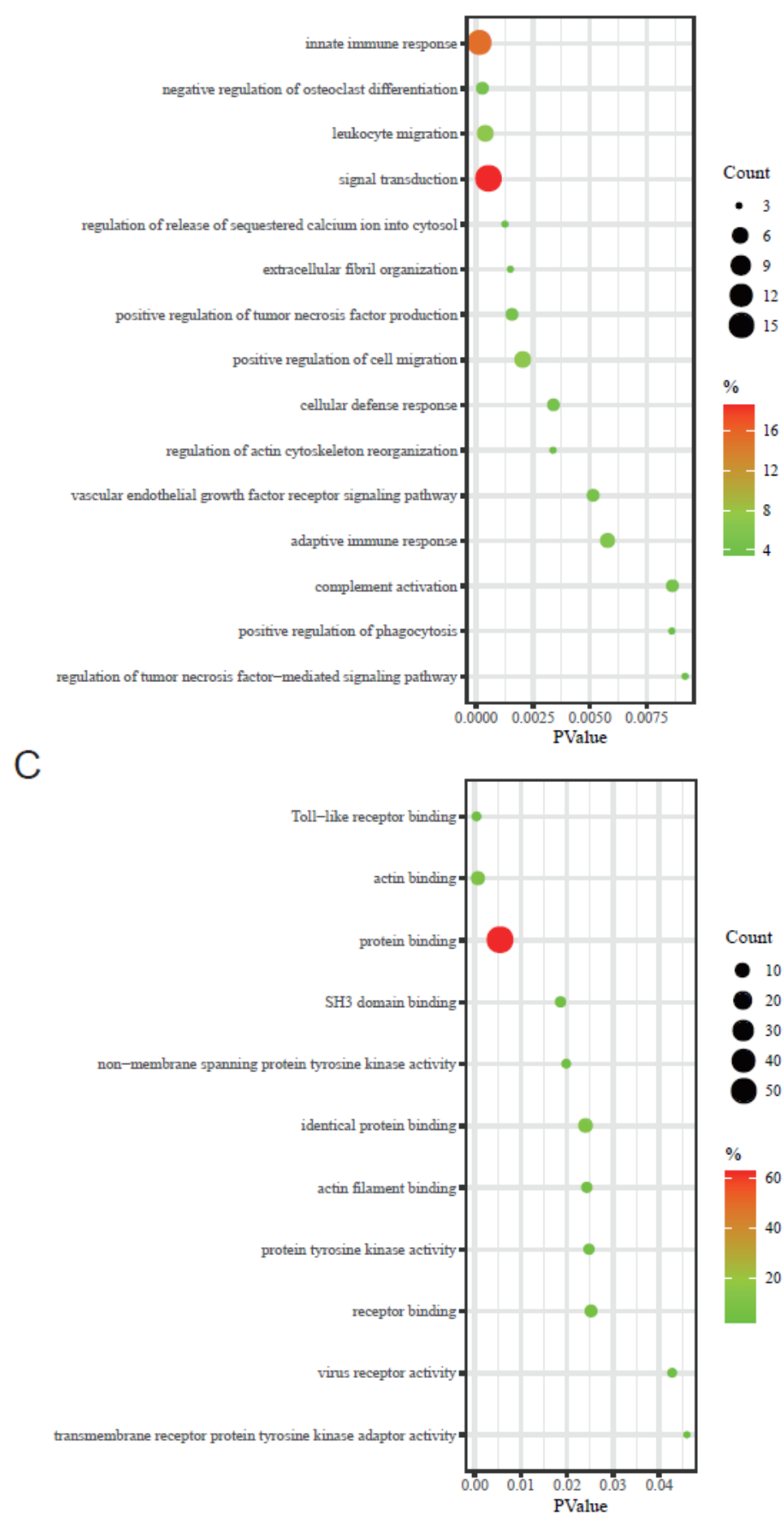

$\mathrm{B}$

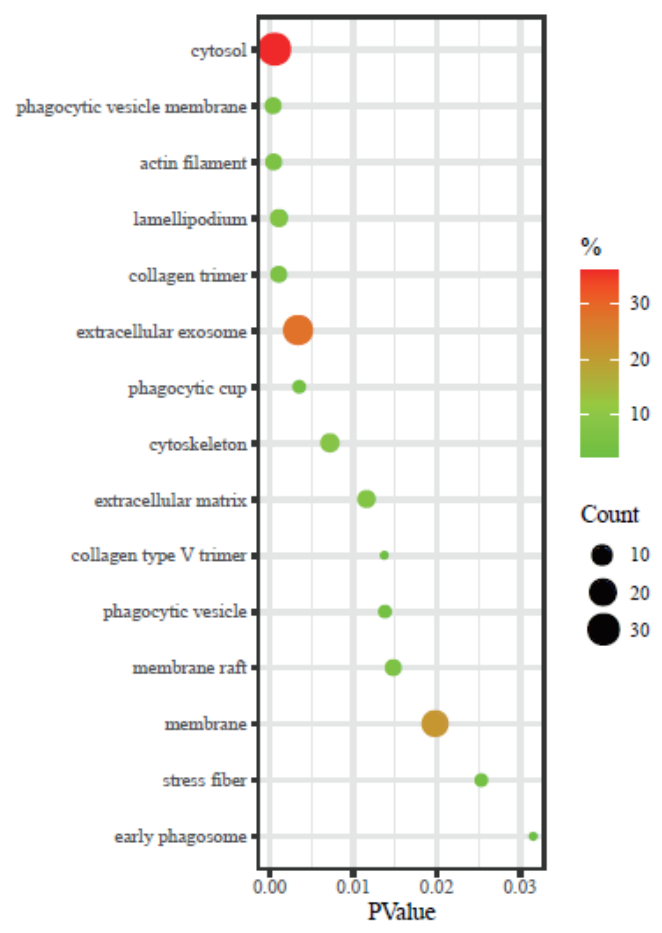

$\mathrm{D}$

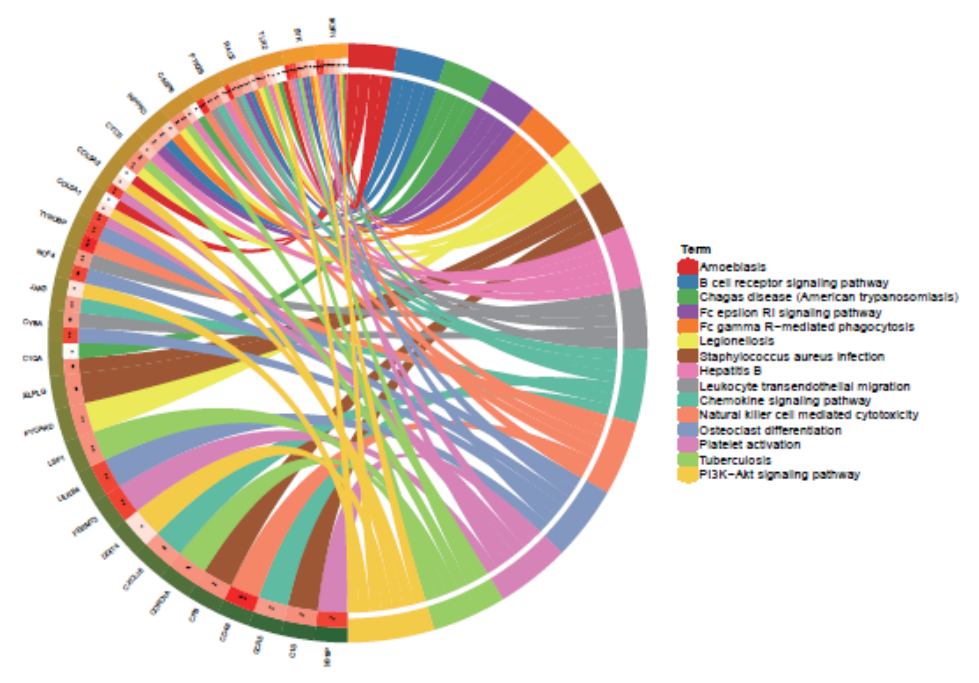

Figure 3

GO and KEGG pathway enrichment analyses. (A)Bubble plot of Homologous DEGs in the term of biological process (BP; TOP15). (B) Bubble plot of Homologous DEGs in the term of cellular component (CC; TOP15). (C) Bubble plot of Homologous DEGs in the term of molecular function (MF; TOP13). (D)Circle plot of KEGG pathway enrichment analysis of Homologous DEGs (TOP15). (Pख0.05) 
A

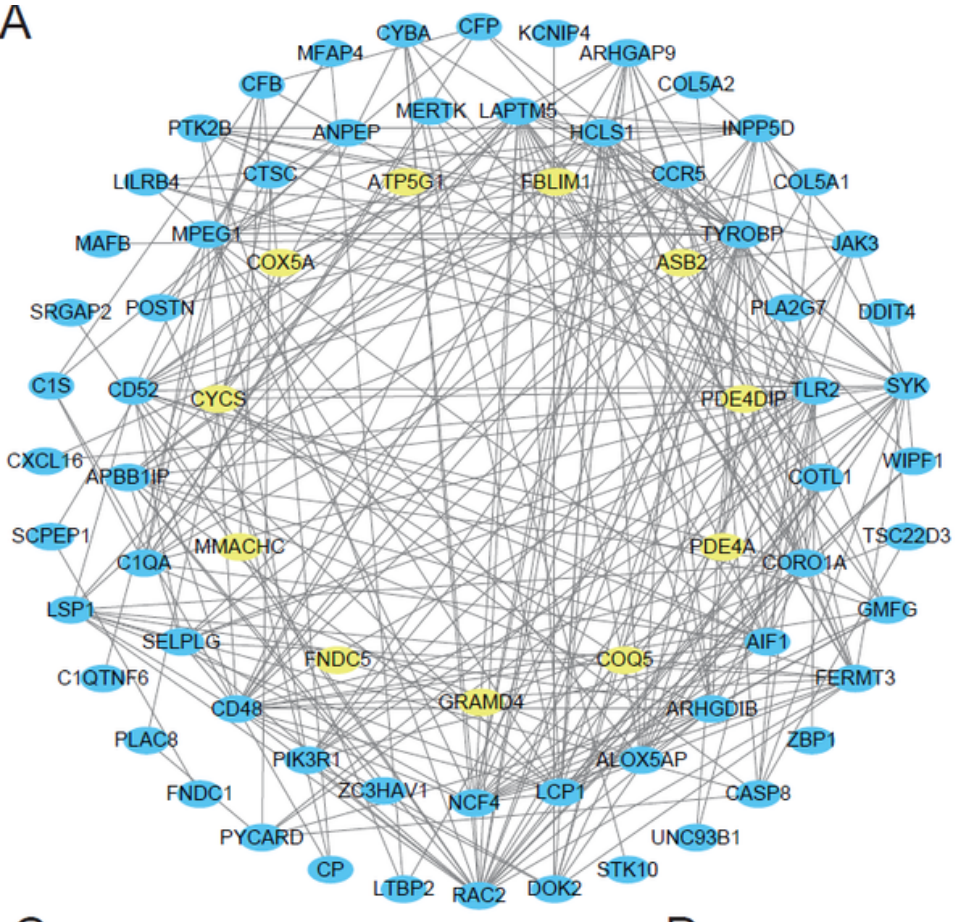

C

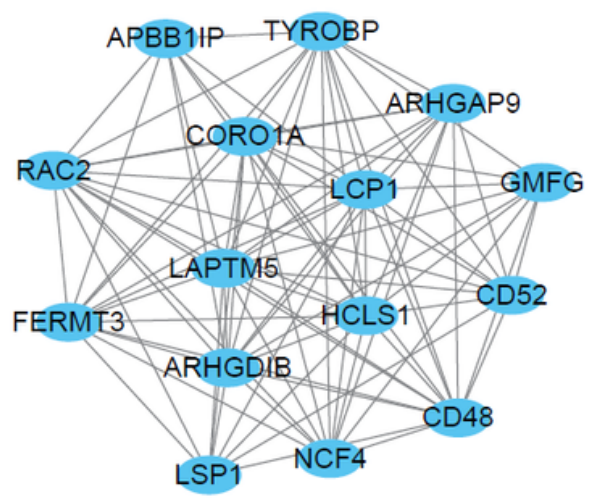

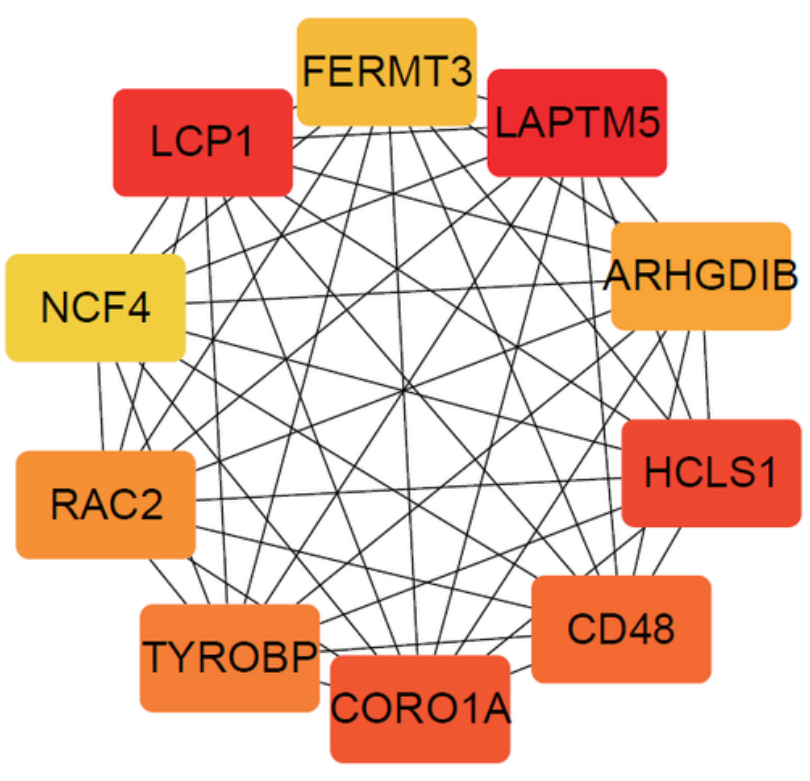

$E$

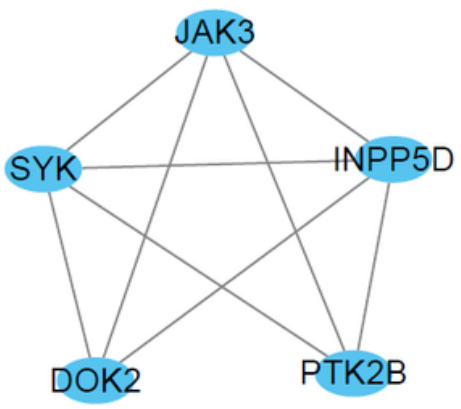

\section{Figure 4}

Protein-Protein Interaction Network, Hub Genes and Key Modules (A) The whole PPI Network. The 11 yellow nodes in the network represent down-regulated genes and the 61 blue nodes in the network represent up-regulated genes. (B) PPI network of the 10 hub genes. (C) PPI network of module 1. (D)PPI network of module 2. (E) PPI network of module 3. 


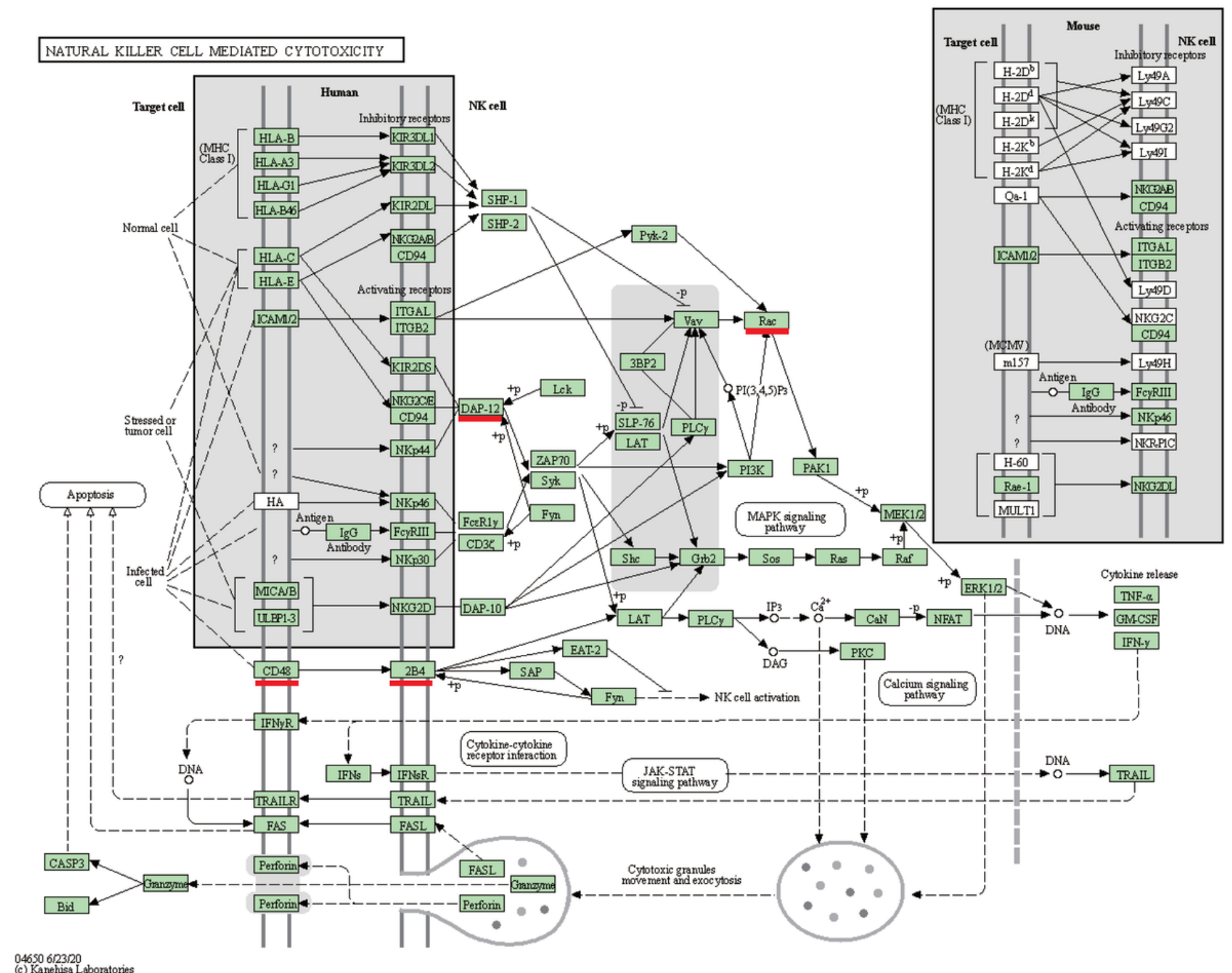

Figure 5

Pathway of Natural killer cell mediated cytotoxicity The Hub gene囚CD48, TYROBP, RAC2囚is highlighted by a subscript red segment. 

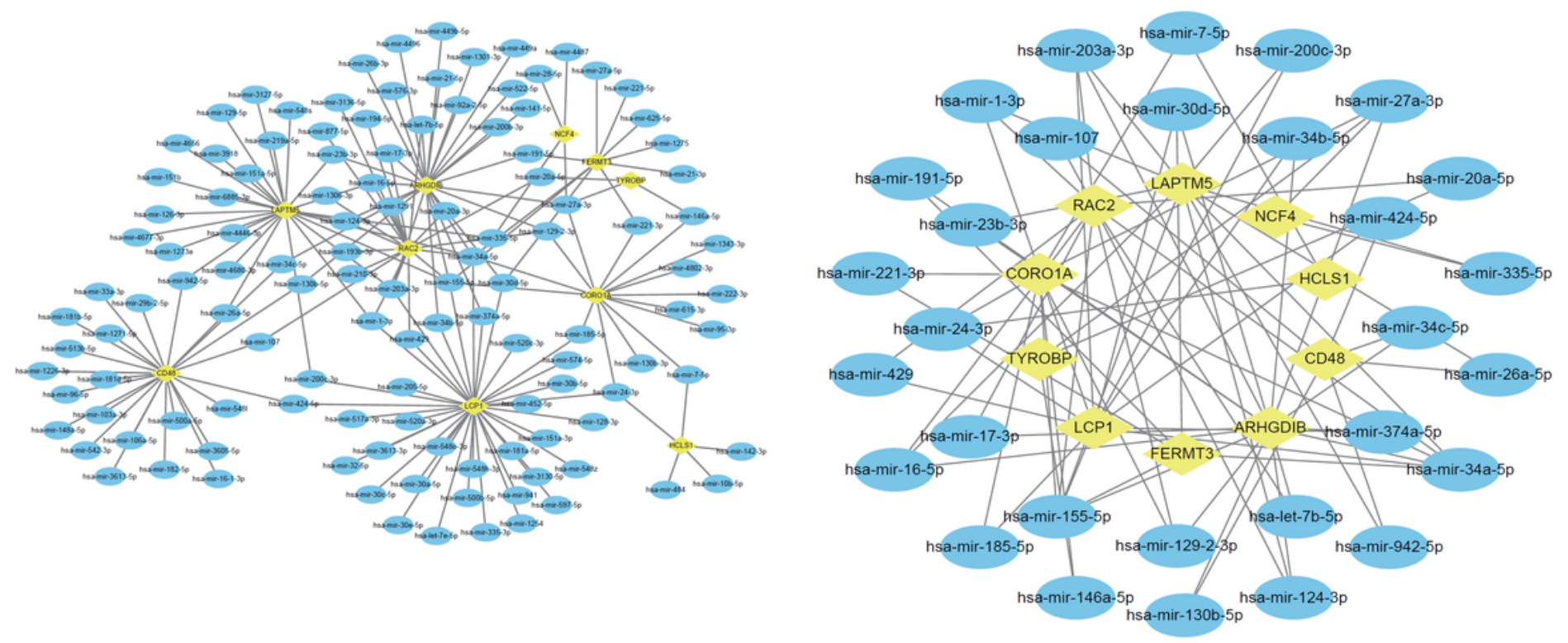

Figure 6

miRNA-mRNA network (A) The whole miRNA-mRNA Network. (B) miRNAs that simultaneously targeted two or more Hub Genes. The yellow diamond nodes represent Hub Genes; The blue elliptic nodes represent miRNAs. 


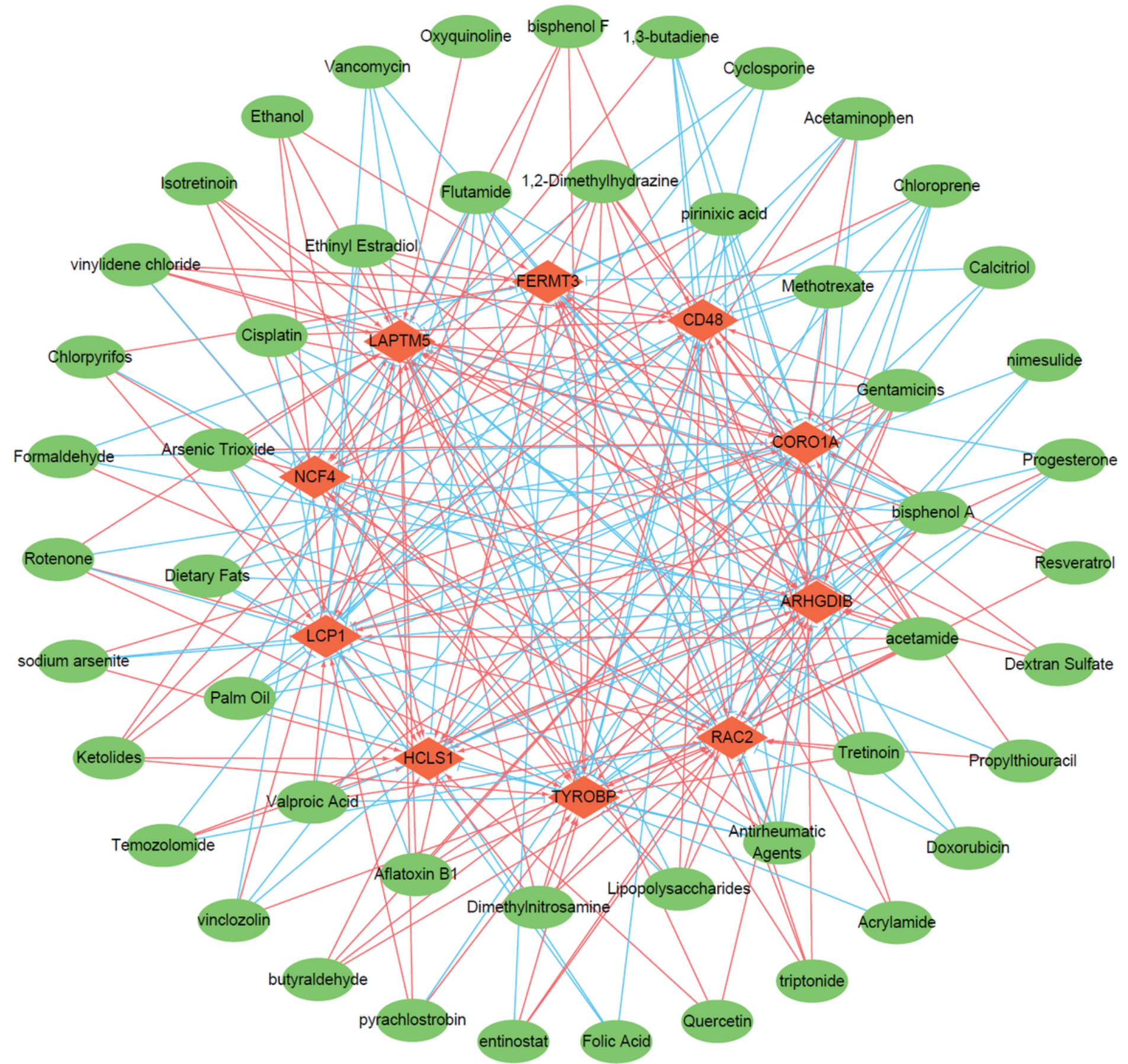

Figure 7

Drug-Hub gene Network The orange-red diamond nodes represent Hub Genes; The green elliptic nodes represent drugs; The orange-red line shows that the drug up-regulates the expression of the target gene; The blue line shows that the drug down-regulates the expression of the target gene;

\section{Supplementary Files}


This is a list of supplementary files associated with this preprint. Click to download.

- Additionalfile1DEGsinGSE84796humanandGSE24088mouse.xIsx

- Additionalfile2Homologousgenesbetweenhumanandmouse.xlsx

- Additionalfile3HomologousDEGs.xlsx

- Additionalfile4FunctionEnrichmentAnalysisofHomologousDEGs.xlsx

- Additionalfile5miRNAsinthemiRNAmRNANetwork.xlsx

- Additionalfile6DrugsthatcouldreduceorincreasetheexpressionlevelsofHubGenes.xlsx

- Additionalfilelegends.docx 